\title{
Prophylactic Antibiotics for Reducing Central Line-associated Blood Stream Infection in Children with Totally Implantable Venous Access Devices
}

Sana Amir Akbar1, Zoona Feroza Qudsia², Muhammad Nabeel Ashraf3, Mansoor Ahmad Tarar4 and Abid Quddus Qazi

${ }^{1}$ Department of Surgery, Shaukat Khanum Memorial Cancer Hospital \& Research Centre, Lahore, Pakistan

2Department of Geriatric Medicine, Prince Phillip Hospital, Llanelli Carmarthenshire, UK

${ }^{3}$ Indus Hospital, Karachi, Pakistan

${ }_{4}$ Department of Surgery, Services Hospital, Lahore, Pakistan

5 Department of Paediatric Surgery, Al Jalile Children's Speciality Hospital, Dubai, UAE

\begin{abstract}
Objective: To analyse the need of prophylactic antibiotic before the insertion of totally implantable venous access devices (TIVADs) in terms of preventing central line-associated blood stream infection (CLABSI) in early postoperative period in pediatric oncology patients.

Study Design: A cohort study.

Place and Duration of Study: Shaukat Khanum Memorial Cancer Hospital and Research Centre, Lahore from January 2005 to June 2016.

Methodology: A total of 645 consecutive children with malignancy, who were implanted with TIVAD, were included in the study. The data were collected retrospectively and divided into two groups on the basis of prophylactic antibiotic administration (Group A) received the antibiotic; and Group B did not receive the antibiotic. Both the groups were compared in terms of positive central blood cultures in the absence of any other clinical source of infection during the early postoperative period of 30 days.

Results: The overall infection rate was $12.54 \%$ (35 out of 279 ) in Group B (did not receive prophylactic antibiotic) and $11.68 \%$ (41 out of 351 ) in Group A (received prophylactic antibiotic) without any significant difference $(p=0.741$ ).

Conclusion: There was no advantage of the use of prophylactic antibiotic before TIVAD insertion in preventing early postoperative CLABSI.
\end{abstract}

Key Words: Prophylactic antibiotic, Venous access, Infection, Central line, Preoperative antibiotics, Children, Pediatric malignancy.

How to cite this article: Akbar SA, Qudsia ZF, Ashraf MN, Tarar MA, Qazi AQ. Prophylactic antibiotics for reducing central line-associated blood stream infection in children with totally implantable venous access devices. J Coll Physicians Surg Pak 2020; 30(3):304-308.

\section{INTRODUCTION}

Reliable, safe and long-term venous access is pivotal for cancer patients as they need frequent administration of intravenous chemotherapeutic agents. ${ }^{1}$ The introduction of totally implantable venous access devices (TIVADs) since early 1980s has revolutionised the approach to venous access, especially in the management of malignant diseases. ${ }^{2}$ These devices are preferred in pediatric oncology patients 3 , who require intermittent long term venous access for chemotherapy. ${ }^{4}$

TIVADs are useful in terms of improved patient's satisfaction ${ }^{8}$ and quality of life, and decreased rate of infection as compared to exterior catheters. . $^{-9}$ So, they are inserted with the aim to last as long as needed without device failure. However, their use is not free of compli-

Correspondence to: Dr. Abid Quddus Qazi, Al Jalile Children's Speciality Hospital, Dubai, UAE

E-mail: abidqazi@me.com

Received: April 03, 2018; Revised: August 07, 2019;

Accepted: September 05, 2019 cations, sometimes resulting in an undesirable outcome of device removal. Central line-associated blood stream infection (CLABSI) is one such complications that may cause interruption in provision of treatment, 10 increased morbidity and increased costs of treatment. 11,12

The use of prophylactic antibiotic before insertion of totally implantable ports is generally not recommended with reference to most of the studies in current literature, as there is no significant decrease in catheter-related infection rates when antibiotic is administered.6,13 However, these studies were done in adult patient population and data is not available for pediatric oncology patients.

So, in this present study, the aim was to analyse whether there is any benefit of a single dose of prophylactic antibiotic before the insertion of TIVADs in terms of preventing early CLABSI in pediatric oncology patients.

\section{METHODOLOGY}

This retrospective cohort study was conducted in the Department of Pediatric Oncology, Shaukat Khanum 
Memorial Cancer Hospital and Research Centre, Lahore, from January 2005 to June 2016, after taking waiver from Institutional Review Board. A total of 645 consecutive patients, who underwent surgical placement of TIVADs for the purpose of chemotherapy administration only, were included. Patients already on antibiotic treat-ment for some other reasons, were excluded from the study. All patients routinely had a complete blood count and prothrombin time / INR were checked prior to the procedure. Catheters were not inserted if the patient was febrile, had clinical evidence of ongoing infection or had neutropenia.

All procedures were performed under general anesthesia by two surgeons, practising in different time periods. Standard sterile techniques were used during placement, and skin was prepared with chlorhexidine. Internal jugular vein was used for placement of venous catheter. Device type and site of placement were similar in both groups. The groups differed in terms of antibiotic administration and technique to approach the vein for cannulation as described later.

One surgeon (from January 2005 to June 2013) routinely used single dose of intravenous antibiotic (amoxicillin/ clavulanic acid, $1200 \mathrm{mg}$ for children $\geq 40 \mathrm{Kg}$ and 30 $\mathrm{mg} / \mathrm{Kg}$ for children $<40 \mathrm{Kg}$ ) within 30 minutes of giving an incision. Moreover, this group had an open venotomy to pass the catheter up to right atrium after trimming to an appropriate length.

Patients in the other group (from September 2013 to June 2016) did not receive prophylactic antibiotic before procedure. In this group, standard Seldinger technique was used for cannulation of vein with the assistance of ultrasound guidance. The catheter was advanced to the right atrium after trimming to an appropriate length.

Rest of the procedure was similar in both groups. A subcutaneous pocket was created with the help of $5 \mathrm{~cm}$ horizontal incision in the anterior chest wall. Tunneling device was used to make a tunnel between the subcutaneous pocket and the lateral part of the neck. The catheter was pulled through the subcutaneous tunnel, flushed with saline, and attached to the port. Port was tucked into the subcutaneous pocket and anchored with sutures. The tip was advanced into the right atrium through the venous opening into the superior vena cava under fluoroscopic guidance. The device was aspirated and flushed. After the procedure, it was filled with heparin and either accessed immediately or left for a later use. Wound was closed in two layers and dressing was applied.

All ports were accessed by a competent nurse, flushed with heparanised saline after every use before removing the access needle and every four weeks, if the device is not in use.

Data regarding patients' age, gender, preoperative absolute neutrophil count and type of primary malig- nancy were retrieved from electronic medical record. The data were divided into two groups on the basis of prophylactic antibiotic administration (Group A) received the antibiotic; and Group B did not receive the antibiotic.

CLABSI was defined as one or more positive blood cultures, where central line was in place for more than two calendar days on the date of event, (with day of device placement being day one) and the line was also in place on the date of event or the day before. Blood culture was ordered only if CLABSI was clinically suspected (fever, chills, leukocytosis or septic shock and no other recognised source of infection) and sampling was done at or around the time of febrile episode prior to starting antibiotics. Blood was drawn simultaneously from the port and a peripheral vein under aseptic conditions in order to obtain paired qualitative blood cultures. Blood cultures from both port sites and peripheral vein must show identical organisms to be labelled as a positive culture.

The data were entered and analysed using SPSS version 20.0. Both the groups were compared in terms of incidence of CLABSI during the early postoperative period of 30 days. Categorical variables, like gender and type of primary malignancy were described as frequencies and percentages. Both the groups were compared by applying Chi-square test. Quantitative variables, like age and preoperative absolute neutrophil counts were described as median and interquartile range (IQR). The groups were compared by applying Mann-Whitney U-test (non-parametric test is applied as the data was not distributed normally). Chi-square test was used to know significant differences in incidence of CLABSI between the two groups; $p$-value of less than 0.05 was taken as statistically significant for all tests. Results were presented in the form of tables.

\section{RESULTS}

During the study period of eleven years and five months, 630 implantable ports were surgically placed in 595 patients. Twenty-five patients $(4.2 \%)$ had device placement for two times and five $(0.84 \%)$ had device placement for three times.

Patients' demographics and preoperative neutrophil count is shown in Table I. There were 398 males $(63.1 \%)$ and 232 females $(36.8 \%)$. Male to female ratio was similar between both groups $(p=0.382)$. Median age was significantly lower in the group that did not receive antibiotic $(p=0.005)$. Median neutrophil count was similar between both the groups $(p=0.640)$.

Patient's diagnosis are shown in Table II and proportion of hematological malignancies between both groups is shown in Table III. Number of patients diagnosed with hematological malignancies were significantly higher in the group not receiving antibiotic $(p=0.007)$. 
Table I: Patients' demographics by treatment groups.

\begin{tabular}{l|l|l|l}
\hline Group & Entire group & Antibiotics (A) & No antibiotics (B) \\
\hline Sample size & 630 & $351(55.7 \%)$ & $279(44.2 \%)$ \\
\hline Gender & & & \\
$\quad$ Male & $398(63.17 \%)$ & $227(64.6 \%)$ & $171(61.2 \%)$ \\
$\quad$ Female & $232(36.8 \%)$ & $124(35.3 \%)$ & $108(38.7 \%)$ \\
\hline Median age (months) & $48($ IQR 60$)$ & $48($ IQR 84$)$ & $36($ IQR 48$)$ \\
\hline Median absolute neutrophil count $\left(\mathrm{x} 10^{3} / \mu \mathrm{l}\right)$ & $3.03(\mathrm{IQR} 3.69)$ & $3.01(\mathrm{IQR} 3.34)$ & $3.07(\mathrm{IQR} 4.09)$ \\
\hline
\end{tabular}

Table II: The various primary malignancies in the study population.

\begin{tabular}{lcc}
\hline Disease & Frequency & Percentage \\
\hline Lymphoma & 142 & 22.5 \\
Leukemia & 141 & 22.4 \\
Sarcoma & 123 & 19.5 \\
Retinoblastoma & 74 & 11.7 \\
Wilm's tumor & 69 & 10.9 \\
Germ cell tumor & 35 & 5.5 \\
Hepatoblastoma & 17 & 2.7 \\
Miscellaneous & 29 & 4.6 \\
\hline
\end{tabular}

The incidence of CLABSI was $12.54 \%$ (35 out of 279 ) in the group that did not receive antibiotic versus $11.68 \%$ (41 out of 351 ) in the group that received antibiotic. Although there is a slight reduction in the incidence of CLABSI when prophylactic antibiotic is used, it was statistically not significant $(p=0.741)$. The most common pathogen identified in blood cultures was staphylococcus aureus followed by klebsiella pneumoniae, gram negative bacilli and yeast species.

Most of the patients with CLABSI in both groups (59 out of 76 or $77.6 \%$ ) were managed conservatively with therapeutic doses of antibiotics. In the rest of the cases (17 out of 76 or $22.36 \%$ ), removal of port was deemed necessary to treat CLABSI and tip culture was obtained after removal. Only four out of seven tips (57\%) turned out to be positive for organisms in the group that did not receive antibiotic as compared to one out of ten (10\%) in the other group ( $p=0.036$, statistically significant).

\section{DISCUSSION}

The current study suggests that prophylactic antibiotic administration before the insertion of TIVADs does not reduce the risk of $C L A B S I$ in the early postoperative period. Moreover, this practice has no statistically significant effect on the incidence of positive tip culture of the removed device. However, small number of ports removed may invalidate the results.

Implantable ports are a reliable source of prolonged venous access in children. ${ }^{3}$ Infection is the most common complication associated with ports, ${ }^{14,15}$ sometimes requiring early removal. 16 According to a survey conducted among fellows of the American College of Surgeons, it was found that more than $88 \%$ of the fellows practise using preoperative antibiotic prophylaxis for central venous access ports placement. 17 This controversial area of clinical practice needs more evidence to weigh the risks and benefits as there are established guidelines for pediatric population suggesting against the use of routine prophylactic antibiotic therapy before insertion of a central venous catheter (NICE guidelines). ${ }^{18}$ However, scarce data is available in literature regarding the role of antibiotics in implantable ports among pediatric patients with oncological problems.

The results of present study replicate the findings of a randomised controlled trial conducted by Karanlik et al. ${ }^{19}$ They studied the rate of early postoperative wound infection among 404 patients (203 received prophylactic cefazolin and 201 received a placebo). They found that the infection rate among antibiotic group was $2.5 \%$ as compared to $3 \%$ in placebo group but the difference was statistically insignificant $(p=0.75)$. The low infection rate in this study can be explained by the fact that this study includes adult population (mean age $=53$ years) and the percentage of hematological malignancies is less as compared to our study.

The results of a meta-analysis comprising of 2,154 patients undergoing TIVAD placement showed that of the 27 infections, $1.39 \%$ occurred in antibiotic prophylaxis group and $1.23 \%$ occurred in nonprophylaxis group. From this, Johnson et al. concluded that the odds ratio of infection (0.85) was not able to show any significant difference in infection rate between the two groups. ${ }^{6}$ In contrast to the above studies, Scaife et al. are of the view that single dose perioperative antibiotic may decrease CLABSI rates. They found that among 459 total patients, all nine $(2 \%)$ infections occurred in the non-prophylactic antibiotic group $(p=0.218) .20$

The present study is different from the above mentioned studies as it is conducted on pediatric oncology patients. As the children are more prone to infections, they could not be compared to adult population, and the clinical decisions for adults cannot be generalised

Table III: Type of malignancy in each group, frequency (percentage).

\begin{tabular}{|c|c|c|c|c|}
\hline Malignancies & Entire group & Antibiotics (A) & No antibiotics (B) & $\mathrm{p}$-value \\
\hline Hematological & $285(45.2)$ & $142(40.5)$ & $143(51.3)$ & 0.007 \\
\hline Non-hematological & $345(54.8)$ & $209(59.5)$ & $136(48.7)$ & \\
\hline
\end{tabular}


to pediatric patients. Current study has a clinical relevance in pediatric oncology patients for deciding about the administration of prophylactic antibiotics before TIVADs insertion. This is also the main strength of this study.

The limitations of this study are that it is a retrospective, single-center study. Moreover, the two study groups are from different points in time so that there is heterogeneity in the study population harbouring various factors that can influence incidence of infection. These factors can be endogenous or exogenous. ${ }^{15}$ In this study groups, factors which were constant and might be unrelated to incidence of infection are preoperative absolute neutrophil count, type of device, targeted vein; and the fact that all insertions were performed under strict aseptic measures in an operating room by experienced surgeons. In addition to this, trained nurses were involved in TIVADs maintenance who followed standard institutional protocols such as barrier precautions and hand hygiene.

Host factors that varied between groups with a statistically significant difference in the present study were age and type of malignancy. The difference in age is clinically not significant as this risk factor is more pronounced in neonatal population, 3,14 and there were no neonates in both the study groups here (age ranging from two months to 18 years). Patients with hematological malignancy are more prone to CLABSI due to immune suppression as compared to patients with solid organ malignancy. 15,19 In this study, significant proportion of patients with hematological malignancy in group $B$ might contribute to increased number of CLABSI in this group. Although neutrophil count at the time of insertion was statistically similar between the groups, it was not recorded at the time of infection.

Two catheter insertion techniques have been described, of which puncturing the vein is better than dissecting it, due to higher infection risk in the later. ${ }^{9}$ This is in contrast to the findings of this study (although statistically insignificant) and can relate to the use of antibiotic prophylaxis and less proportion of patients with hematological malignancies in this group (group A). All these factors point out to the need of a prospective randomised trial with adequate sample size to find the role of prophylactic antibiotic for TIVAD placement in pediatric oncology patients.

\section{CONCLUSION}

There was no added advantage of the use of prophylactic antibiotic before TIVAD insertion in preventing early postoperative CLABSI.

\section{ETHICAL APPROVAL:}

Study was conducted after obtaining approval from the
Institutional Review Board.

\section{PATIENTS' CONSENT:}

Consent for the participation in study was not obtained from patients as data was collected from medical record without disclosing the indentity of participants.

\section{CONFLICT OF INTEREST:}

Authors declared no conflict of interest.

\section{AUTHORS' CONTRIBUTION:}

SAA: Research design, literature search, data collection and analysis, result interpretation, manuscript writing, final manuscript preparation and proofreading, overall coordination.

ZFQ, MNA, MAT: Data collection and drafting manuscript. AQQ: Substantial contribution to overall guidance, research design and study conception, result interpretation and discussion, supervised study, proofreading of manuscript and revised critically for important intellectual content, final approval.

All authors approved final version of the manuscript.

\section{REFERENCES}

1. Kreidieh FY, Moukadem HA, El Saghir NS. Overview, prevention and management of chemotherapy extravasation. World J Clin Oncol 2016; 7:87-97.

2. El-Balat A, Schmeil I, Karn T, Holtrich U, Mavrova-Risteska L, Rody A, et al. Catheter-related complications of subcutaneous implantable venous access devices in breast cancer patients. In Vivo 2018; 32:1275-81.

3. Larson SD, Mancini MC. Vascular access in children. Medscape.

4. D'Souza PC, Kumar S, Kakaria A, Al-Sukaiti R, Zahid KF, Furrukh $\mathrm{M}$, et al. Use of port-a-cath in cancer patients: A single-center experience. J Infect Dev Ctries 2014; 8:1476-82.

5. Füreder $T$, Mähr B, Berghoff AS, Heynar $H$, Dressler A, Zielinski CC, et al. A cross-sectional study of patients' satisfaction with totally implanted access ports. Clin J Oncol Nurs 2016; 20:175-80.

6. Johnson E, Babb J, Sridhar D. Routine antibiotic prophylaxis for totally implantable venous access device placement: Metaanalysis of 2,154 patients. J Vasc Interv Radiol 2016; 27:339-43.

7. Patel GS, Jain K, Kumar R, Strickland AH, Pellegrini L, Slavotinek $\mathrm{J}$, et al. Comparison of peripherally inserted central venous catheters (PICC) versus subcutaneously implanted port-chamber catheters by complication and cost for patients receiving chemotherapy for non-haematological malignancies. Support Care Cancer 2014; 22:121-8.

8. Singh KR, Agarwal G, Nanda G, Chand G, Mishra A, Agarwal $A$, et al. Morbidity of chemotherapy administration and satisfaction in breast cancer patients: A comparative study of totally implantable venous access device (TIVAD) versus peripheral venous access usage. World J Surg 2014; 38:108492.

9. Zerati AE, Wolosker N, de Luccia N, Puech-Leão P. Totally implantable venous catheters: History, implantation technique and complications. J Vasc Bras 2017; 16:128-39. 
10. Merle V, Marini H, Di Fiore F, Lottin M, Gray C, Loeb A, et al. Morbidity-mortality conference for adverse events associated with totally implanted venous access for cancer chemotherapy. Support Care Cancer 2016; 24:1857-63.

11. Werner $\mathrm{E}$. It is time to address ambulatory central venous line infections in pediatric hematology/oncology patients. Pediatr Blood Cancer 2017; 64:221-2.

12. Raad I, Chaftari AM. Advances in prevention and management of central line-associated bloodstream infections in patients with cancer. Clin Infect Dis 2014; 59:S340-3.

13. Jo JE, Tang EY, Pua BB. The role of antibiotics in preventing totally implantable venous access device (TIVAD) infections: Is there a population that would benefit? Clin Imag 2018; 51: 213-6.

14. Ullman AJ, Marsh N, Mihala G, Cooke M, Rickard CM. Complications of central venous access devices: A systematic review. Pediatrics 2015; 136:1331-44.

15. Aparna S, Ramesh S, Appaji L, Srivatsa K, Shankar G, Jadhav V, et al. Complications of chemoport in children with cancer: Experience of 54,100 catheter days from a tertiary cancer center of Southern India. South Asian J Cancer 2015; 4:143.

16. Loh $\mathrm{AH}$, Chui $\mathrm{CH}$. Port-A-Cath insertions in acute leukaemia and childhood malignancies. Asian J Surg 2007; 30:193-9.

17. Nelson ET, Gross ME, Mone MC, Hansen HJ, Nelson EW, Scaife CL. A survey of American College of Surgery fellows evaluating their use of antibiotic prophylaxis in the placement of subcutaneously implanted central venous access ports. Am J Surg 2013; 206:1034-40.

18. Guidelines and Audit Implementation Network (GAIN). Guidelines for insertion and maintenance of central venous access devices in children and young people 2012. https://www.rqia. org.uk/RQIA/files/cb/cb2bc4e1-f98a-4965-9489-85bdea f6fc0d.pdf (Accessed on February 6, 2018).

19. Karanlik H, Kurul S, Saip P, Unal ES, Sen F, Disci R, et al. The role of antibiotic prophylaxis in totally implantable venous access device placement: Results of a single-center prospective randomized trial. Am J Surg 2011; 202:10-15.

20. Scaife CL, Gross ME, Mone MC, Hansen HJ, Litz CL, Nelson ET, et al. Antibiotic prophylaxis in the placement of totally implanted central venous access ports. Am J Surg 2010; 200: 719-23. 